\title{
The mechanism of pentabromopseudilin inhibition of myosin motor activity
}

Roman Fedorov ${ }^{1}$, Markus Böhl' ${ }^{2}$, Georgios Tsiavaliaris ${ }^{3}$, Falk K. Hartmann ${ }^{3}$, Manuel H. Taft ${ }^{3}$, Petra Baruch ${ }^{1}$, Bernhard Brenner ${ }^{4}$, René Martin ${ }^{5}$, Hans-Joachim Knölker ${ }^{5}$, Herwig O. Gutzeit ${ }^{2}$ \& Dietmar J. Manstein ${ }^{1,3^{*}}$

${ }^{1}$ Research Centre for Structure Analysis, OE8830, Hannover Medical School, 30623 Hannover, Germany. ${ }^{2}$ Department of Biology, TU Dresden, Zellescher Weg 20b, 01217 Dresden. ${ }^{3}$ Institute for Biophysical Chemistry, OE4350, Hannover Medical School, 30623 Hannover, Germany. ${ }^{4}$ Institute for Molecular and Cellular Physiology, OE4210, Hannover Medical School, 30623 Hannover, Germany. ${ }^{5}$ Department of Chemistry, TU Dresden, Bergstrasse 66, 01069 Dresden, Germany.

Correspondence should be addressed to DJM (manstein@bpc.mh-hannover.de).

We identified pentabromopseudilin (PBP) as potent inhibitor of myosindependent processes such as isometric tension development and unloaded shortening velocity. PBP-induced reductions in the rate constants for ATPbinding, ATP-hydrolysis and ADP dissociation extend the time required per myosin ATPase cycle in the absence and presence of actin. Additionally, coupling between the actin and nucleotide binding sites is reduced in the presence of the inhibitor. The selectivity of PBP differs from that observed with other myosin inhibitors. To elucidate the binding mode of PBP, we crystallized the Dictyostelium myosin-2 motor domain in the presence of $\mathrm{Mg}^{2+} \mathrm{ADP}-m e t a-$ vanadate and PBP. The electron density for PBP is unambiguous and shows PBP to bind at a novel allosteric site near the tip of the 50-kDa domain, at a distance of $16 \AA$ from the nucleotide binding site and $7.5 \AA$ away from the blebbistatin binding pocket.

Motor proteins belonging to the myosin family are amongst the best understood molecular machines. Myosin motor domains are accessible for analysis by an exceptionally wide range of experimental approaches, as they produce chemical, mechanical, and spectroscopic changes during their active cycle. As a result, our understanding of the actomyosin system has now advanced to include detailed insights into the molecular mechanisms underlying the conversion of chemical energy into force and movement, which is catalyzed by the myosin motor domain. However, many questions remain to be solved for the majority of the myosin family members. Their specific cellular functions and potential involvement in disease processes remain to be elucidated for many of the 39 members of the myosin family produced by the human body. What is evident already is that the role of myosins extends far beyond muscle contraction and the intracellular movement of vesicles. Their activities include roles in endocytosis, cytokinesis, nuclear anchoring, RNA polymerase I-dependent transcription, sensitization of transduction channels, and several members of the myosin family carry domains that are directly involved in signal transduction ${ }^{1-6}$.

Small chemical compounds that selectively alter the motor activity of individual myosin isoforms, hold strong potential as tools for the analysis of complex biological processes and as therapeutics. In the process of performing assays aiming at the modulation of the $\beta$ adrenergic response of cardiomyocytes, we noticed that the marine natural product pentabromopseudilin (PBP) inhibits myosin-dependent processes. The systematic name of PBP is 2,3,4-tribromo-5-(3,5-dibromo-2-hydroxyphenyl)- $1 H$-pyrrole. PBP was originally isolated as an antibiotic from Pseudomonas bromoutilis with antitumor and phytotoxic activities and was shown to inhibit both 12- and 15-human lipoxygenase ${ }^{7,8}$.

Further experiments using purified myosin revealed that PBP acts as a potent and reversible inhibitor of myosin ATPase and motor activities. To elucidate the inhibitory mechanism and to identify the PBP binding site, we characterized the effect of PBP on the kinetics of the ATPase cycle of myosins from several classes and organisms, examined its effect on the contraction of muscle fibers, and solved the crystal structure of PBP bound to the 
Dictyostelium discoideum myosin-2 motor domain. The structure shows the inhibitor to bind in a pocket close to the actin binding region at the tip of the 50-kDa domain and indicates myosin-isoform specific differences in the extent of PBP mediated inhibition. Molecular modeling studies, predicting PBP to display particularly high affinity for vertebrate myosin$5 \mathrm{a}$, are confirmed by the results of in vitro motility and ATPase assays. The selective inhibition of a myosin- 5 dependent process in yeast shows the compound's potential as a tool in cell biological applications.

\section{RESULTS}

\section{Kinetic mechanism of PBP-mediated inhibition of myosin}

PBP inhibits the ATPase activities of a wide range of myosins with a distinct pattern of selectivity. Here, we describe in detail the effects of PBP on the kinetic behavior of $D$.

discoideum $(D d)$ myosin-5b. A wide range of spectroscopic signals can be measured with this myosin in the presence of PBP and its kinetic properties have been described in detail ${ }^{9}$.To show the effect of PBP on the functional properties of other myosins, we include selected results for rabbit skeletal muscle HMM, chicken myosin-5a, and recombinant $D d$ class- 1 and 2 myosins.

PBP decreases the maximum rate of basal and actin-activated myosin ATPase of all myosins tested. Values for the rate of maximum ATP turnover $\left(k_{\text {cat }}\right)$, the concentration of Factin at which half-maximal activation is achieved $\left(K_{\text {app }}\right)$, and the apparent second order rate constant for actin binding $\left(k_{\mathrm{cat}} / K_{\mathrm{app}}=\Gamma\right)$ were obtained from plots of steady-state actinactivated ATPase activity against the concentration of F-actin (Fig. 1a). $\Gamma$ is a direct measure of the coupling efficiency between the actin and nucleotide binding sites. Plots of steady-state actin-activated ATPase activity against the concentration of PBP could be fitted to sigmoidal curves for each myosin (Fig. 1b). The observed level of inhibition was greater than 50-fold for all myosins tested at saturating PBP concentrations. Amongst the myosins tested, chicken myosin-5a displays the most potent inhibition of actomyosin ATPase activity by PBP with an $\mathrm{IC}_{50}$ of $1.2 \mu \mathrm{M}$. Additionally, chicken myosin-5a is the only myosin that displays an apparent dissociation equilibrium constant for actin binding in the presence of ATP that is considerably lower than the maximum actin concentrations accessible $\left(K_{\text {app }}=1.4 \mu \mathrm{M}\right)$. Therefore, the $\mathrm{IC}_{50}$ values in the presence of actin shown in Table 1 define lower estimates for the other myosins. A sigmoidal dependence on the concentration of PBP was also observed for basal ATPase activity. Most myosins tested displayed 2 to 3 -fold lower $\mathrm{IC}_{50}$ values in the absence of actin than in its presence (Table 1).

To determine the exact mode of action of PBP, transient kinetic measurements were performed with $D d$ myosin-5b. Results were interpreted according to the scheme shown in Figure 1 that describes mechanisms for the PBP-mediated inhibition of basal and actinactivated myosin ATPase activity. The major transient kinetics results obtained for $D d$ myosin- $5 \mathrm{~b}$ are summarized in Table 1 . We used mantATP and mantADP for the initial characterization of the effect of PBP on nucleotide binding ${ }^{10}$. Addition of PBP to myosin motor domain constructs led to a marked loss in the rate and the amplitude of the mantATPinduced signal change. The observed transients showed monophasic behavior in the absence of PBP and in the presence of saturating concentrations of the inhibitor. At intermediate concentrations biphasic behavior was observed, where the fast phase corresponds to binding of mantATP to M and the slow phase to mantATP binding to IM (Fig. 1c). The change in amplitude of the fast phase with increasing PBP concentrations shows an inverse hyperbolic dependence and gives an $\mathrm{IC}_{50}$ value of approximately $15 \mu \mathrm{M}$ (Fig. 1c, insert). The presence of $50 \mu \mathrm{M}$ PBP leads to an approximately 3 -fold reduction in the apparent second order rate constants for mantATP binding from $0.85 \mu \mathrm{M}^{-1} \mathrm{~s}^{-1}$ to $0.29 \mu \mathrm{M}^{-1} \mathrm{~s}^{-1}\left(k_{+1}, k_{+1 \mathrm{i}}\right)$, while a less than 2-fold decrease from 0.17 to $0.11 \mu \mathrm{M}^{-1} \mathrm{~s}^{-1}$ is observed for mantADP binding $\left(k_{-4}, k_{-4 \mathrm{i}}\right)$

(Supplemental Fig. 1a,b online). The rate of mantADP release is increased from $0.92\left(k_{+4}\right)$ to $1.60 \mathrm{~s}^{-1}\left(k_{+4 i}\right)$. The calculated dissociation equilibrium constants $K_{4}(5.4 \mu \mathrm{M})$ and $K_{4 \mathrm{i}}(14.5$ $\mu \mathrm{M})$ indicate a 3 -fold reduction in the affinity of $D d$ myosin-5b for ADP in the presence of PBP (Table 1). 
To determine rates of ATP binding and hydrolysis, we used the time-dependent change in the intrinsic protein fluorescence that follows the addition of excess ATP to $D d$ myosin-5b. The maximum rate of ATP hydrolysis $\left(k_{+2}+k_{-2}\right)$ is $350 \mathrm{~s} \mathrm{~s}^{-1}$ for $D d$ myosin-5b. The addition of $25 \mu \mathrm{M}$ PBP leads to a 10-fold reduction in the rate of ATP hydrolysis and the apparent second-order rate constant for ATP binding is slowed from $0.475 \mu \mathrm{M}^{-1} \mathrm{~s}^{-1}\left(K_{1} k_{+\mathrm{c}}\right)$ to 0.033 $\mu \mathrm{M}^{-1} \mathrm{~s}^{-1}\left(K_{1 i} K_{+\mathrm{ci}}\right)$ (Fig. 1d). To gain more information about the steps affected by PBP binding, we performed single turnover experiments with $D d$ myosin-5b using mantATP as substrate (Supplemental Fig. 1c online). In the absence of PBP the time evolution of the reaction can be separated in three phases: an initial rise in mant-fluorescence corresponding to binding of mantATP, a short plateau phase determined by the rate of the conformational change that follows mantATP binding, and a drop in mant-fluorescence corresponding to release and rebinding of mantADP $\left(k_{-4}+k_{+4}\right)$. All three phases are extended in the presence of PBP. We interpret the extension of the plateau phase in the presence of PBP as the consequence of both slower mantATP hydrolysis and increased mantADP affinity. Single and multiple turnover experiments using ATP as substrate and measuring the release of inorganic phosphate ${ }^{11}\left(\mathrm{P}_{\mathrm{i}}\right)$ indicate that that $P_{\mathrm{i}}$ release is the rate limiting step for $D d$ myosin-5b basal ATPase activity. Under multiple turnover conditions, ADP release becomes the rate limiting step

(Supplemental Fig. 1d online). In the presence of PBP, actin appears to increase the affinity of $D d$ myosin- $5 \mathrm{~b}$ for ADP rather than to reduce it, while $\mathrm{P}_{\mathrm{i}}$ release is not greatly affected (Table 1).

The rate of ATP binding to pyrene-labeled actomyosin was monitored by observing the exponential $20 \%$ increase in pyrene fluorescence, as the complex dissociates upon addition of excess ATP. At lower ATP concentrations and in the absence of PBP the observed rate constants increased linearly up to $50 \mu \mathrm{M}$ ATP, giving a second order rate binding constant of $0.19 \mu \mathrm{M}^{-1} \mathrm{~s}^{-1}\left(\mathbf{K}_{\mathbf{1}} \mathbf{K}_{+6}{ }^{\prime}\right)$. Initial experiments showed only minor PBP-induced changes in the rate of the ATP-induced dissociation for the complex. This led to the question whether ATPinduced dissociation is more potently inhibited by PBP in the presence of ADP. Preincubation of the actomyosin complex with PBP and $5 \mu \mathrm{M}$ ATP followed by aging of the reaction mixture to allow formation of the IAMD complex, did indeed lead to a larger drop in the observed rates of ATP-induced dissociation of the complex. Due to the presence of nucleotide-free and ADP-bound states under these conditions, the reaction shows biphasic behavior both in the absence and the presence of $50 \mu \mathrm{M}$ PBP (Supplemental Fig. 1e online). The initial fast phase corresponds to the binding of ATP to the nucleotide-free complex and the second phase is determined by the dissociation of ADP from the AMD or IAMD complex. PBP affects the amplitudes and rate constants observed for both phases. Analysis of the fast phase indicates a $30 \%$ reduction in the second order rate constant for ATP binding to IAM $\left(\mathbf{K}_{\mathbf{1} \mathbf{i}} \mathbf{K}_{-6 \mathbf{i}}\right)$ to $0.13 \mu \mathrm{M}^{-1} \mathrm{~s}^{-1}$. Addition of high ADP concentrations leads to monophasic dissociation reactions, with the observed rates of ATP-induced dissociation directly corresponding to the ADP dissociation rate from AMD $\left(\mathbf{k}_{+4},=17.4 \mathrm{~s}^{-1}\right)$ or IAMD $\left(\mathbf{k}_{+4 \mathrm{i}}=7.0 \mathrm{~s}^{-}\right.$ $\left.{ }^{1}\right)$ (Supplemental Fig. 1f online). The total amplitude of the change in pyrene fluorescence is not affected by the presence of PBP, indicating that PBP does not prevent the formation of the strongly bound state.

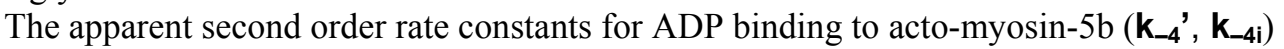
were determined by monitoring the increase in mant-fluorescence upon the addition of increasing concentrations of the fluorescent analogue to acto-myosin- $5 \mathrm{~b}$. The observed rate constants for the exponential increase in fluorescence were linearly dependent on [mantADP] in the range from 1 to $30 \mu \mathrm{M}$ (data not shown). The slopes of the straight lines fitted to the data gave values of 4.0 and $5.7 \mu \mathrm{M}^{-1} \mathrm{~s}^{-1}$ for $\mathbf{k}_{-4}$ ' and $\mathbf{k}_{-4 \mathrm{i}}$, respectively. The dissociation equilibrium constant for mantADP binding to acto-myosin-5b in the presence of PBP $\left(\mathbf{K}_{4 \mathrm{i}}\right)$ was calculated from $\mathbf{k}_{+4 i} / \mathbf{k}_{-4 i}$ and corresponds to $1.23 \mu \mathrm{M}$. In the presence of $50 \mu \mathrm{M}$ PBP, actin increases the affinity of ADP for $D d$ myosin-5b more than 12 -fold as indicated by the ratio of $K_{4 i} / K_{4 i}$ (Table 1). To demonstrate that the inhibitor exercises an allosteric effect on the open-closed transition of the nucleotide binding pocket, we used a $D d$ myosin-2 motor domain construct retaining a single tryptophan residue at position $501(\operatorname{Trp} 501)^{12}$. The 
fluorescence emission intensity from Trp501, located at the distal end of the relay helix, is very sensitive to the open-closed transition of the nucleotide binding pocket ${ }^{13}$. (Supplemental Figure 2 online).

\section{PBP-mediated inhibition of myosin motor activity}

To directly examine the inhibition of myosin motor activity by PBP, we performed in vitro motility assays and examined the effect of the inhibitor on the contraction of skinned muscle fibers. The in vitro motility assays described here were performed with chicken myosin-5a, which displays the full extent of inhibition at PBP concentrations below $10 \mu \mathrm{M}$ in the in vitro motility assay. Under standard assay conditions, chicken myosin-5a moves F-actin with an average velocity of $600 \mathrm{~nm} \mathrm{~s}^{-1}$. Addition of $10 \mu \mathrm{M}$ PBP reduced the average sliding velocity approximately 50 -fold to $13 \mathrm{~nm} \mathrm{~s}^{-1}$, without reduction in the number of moving filaments (Fig. 1e). These results show that myosin heads are still able to interact productively with Factin in the presence of PBP, albeit at strongly reduced rates. PBP-mediated inhibition is reversible, as motile activity is almost completely recovered within minutes after wash-out of the inhibitor.

Next, we examined the ability of PBP to decrease tension generation by actively contracting fast skeletal muscle fibers. In the presence of $100 \mu \mathrm{M}$ PBP, we observed a 3-fold reduction in the steady-state level of isometric tension generated by skinned fibers of rabbit psoas muscle. The steady state level of isometric tension is shown as a function of the PBP concentration in Figure 1f. The apparent $\mathrm{IC}_{50}$, obtained from a sigmoidal fit to the data, corresponds to $25 \mu \mathrm{M}$ for this skeletal muscle myosin-2 dependent process. Large PBPmediated effects are observed for the velocity of unloaded shortening. Maximum inhibition was reached after equilibration of fibers for 2 hours in relaxing solution with $200 \mu \mathrm{M}$ PBP. Superposition of unloaded shortening at different degrees of $\mathrm{Ca}^{2+}$-activation and in presence of different inhibitor-concentrations reveals that in the presence of inhibitor unloaded shortening is slowed down both in the initial and late phases of shortening (Fig. 1g). The effects of PBP on tension redevelopment and stiffness-speed profiles ${ }^{14}$ are shown in

(Supplemental Figure 3 online). The observed PBP-induced reductions in $k_{\mathrm{TR}}$ and isometric force, minor changes in stiffness, and slower shortening during the late phase are consistent with a slower transition into force-generating states. A slow initial phase of shortening is consistent with the observed reductions in the rates for ADP-release and ATP-induced dissociation in the presence of PBP.

\section{Identification of the PBP-binding site}

To identify the PBP-binding site, we crystallized the $D d$ myosin- 2 motor domain $\mathrm{Mg}^{2+} \mathrm{ADP}-$ meta-vanadate complex in the absence and presence of PBP. We solved the structures by molecular replacement and refined them to $2.3 \AA$ and $2.8 \AA$ resolution, respectively. The overall fold of the motor domain in both structures is similar to that reported for the pre-powerstroke conformation $^{15}$. In contrast to the geometries of the ortho-vanadate-ADP complexes, where the vanadate moiety has a trigonal bipyramidal conformation, the meta-vanadate complex shows tetrahedral symmetry and the distance between the vanadium atom and the $\beta-\gamma$-bridging oxygen is $1.8 \AA$ instead of $2.1 \AA$. The meta-vanadate ion thus fully replaces the position of $\gamma$ phosphate forming a covalent bond with the $\beta$-phosphate moiety of ADP (Fig. 2a). Therefore, the nucleotide meta-vanadate complex at the active site and the overall myosin motor domain structure resemble most closely those of ATP and the ATP-bound state.

The electron density for PBP is unambiguous and shows the inhibitor to bind in a pocket close to actin-binding residues and located $16 \AA$ away from the nucleotide binding site. PBP is in a conformation where the phenyl and pyrrole ring systems are bent by $12^{\circ}$ out of plane and twisted by $20^{\circ}$ against each other (Fig. 2b). The total protein surface area in contact with PBP comprises $255 \AA^{2}$ and both polar and apolar residues contribute to the binding of PBP. The inner face of this PBP-binding pocket is formed by helix-13 (Lys265-Val268) and helix-21 (Val411-Leu441) from the 50kDa upper domain (U50). The outer face is contributed by the strut loop (Asn588-Gln593) and loop 2 (Asp614-Thr629), which are connected by the helix- 
loop-helix motif that covers parts of the cleft and the U50 in a lid-like fashion. The PBPbinding pocket has a large opening towards the exterior and the large cleft that is surrounded by residues from loop-2, helix-21, helix-29 (Val630-Glu646), and the loop connecting $\beta 7$ (Ile253-Leu261) with helix-13. Additionally, a smaller access channel is located between loop-2 and the strut loop. The $\alpha$-carbon atoms in the $\mathrm{Mg}^{2+} \mathrm{ADP}-m e t a$-vanadate complex structures with and without bound PBP superimpose with an r.m.s. deviation of $0.695 \AA$, indicating that the myosin motor domain does not undergo any major conformational changes upon binding of PBP, except for local changes directly involved in the coordination of the inhibitor. The importance of loop-2 and the strut loop for coupling between the actin und nucleotide binding sites and cleft closure were demonstrated by mutagenesis studies ${ }^{13}$. In particular it was shown that removal or repositioning of Asp590, a residue that is directly involved in the coordination of PBP, destroys strong binding of Dictyostelium myosin-2 to F$\operatorname{actin}^{16}$.

Binding of PBP requires several rearrangements of residues inside and near the binding pocket as described in Supplemental Figure 4 online. Based on the structure of the $D d$ myosin-2 motor domain with bound PBP, we performed molecular modeling studies to elucidate the structural basis of the observed differences in PBP-binding to myosins from different classes. The results for the modeling of the complexes formed with class- 1 and class-5 myosins are shown in Figure 3. The interaction of a basic residue corresponding to Lys265 in myosin-2 with the hydroxyl group of PBP is a common feature of all complexes. However, the total number of interactions and their strength is predicted to vary considerably between individual myosins. $D d$ myosin- 2 forms four hydrogen bonds and six weaker vander-Waals interactions with PBP (4/6). The numbers of predicted interactions with PBP are 12 (6/6) for chicken myosin-5a, 8 (6/2) for Sc Myo2p, 9 (5/4) for $D d$ myosin-5b, and 6 (3/3) for $D d$ myosin-1E. In agreement with these results the steady-state ATPase of chicken myosin5a displayed a much larger decrease in ATPase activity than observed for the other myosins. Additionally, the predicted number of interactions correlates well with the $\mathrm{IC}_{50}$ shown in Table 1.

\section{Inhibition of myosin-5-dependent cellular processes}

To test the specific inhibition of myosin- 5 function in a cellular context, we exposed Saccharomyces cerevisiae cells to concentrations of 100 to $500 \mathrm{nM}$ PBP. S. cerevisiae (Sc) has five myosin heavy chain genes encoding a class-2 myosin (Myolp), two class-1 myosins (Myo3p, Myo5p), and the class-5 myosins Myo2p and Myo4p. Myo2p plays a crucial role in polarized distribution of mitochondria and is required for retention of newly inherited mitochondria in yeast cells during cell division ${ }^{17,18}$. Myo4p is required for mRNA transport and facilitates movement of ER tubules into the growing bud ${ }^{19}$. Myo4p null mutants are viable and display no detectable phenotype ${ }^{20}$. Deletion of the $m y o 3$ and myo5 genes, encoding class-1 myosins, leads to severe defects in growth and actin cytoskeletal organization ${ }^{21}$.

Severe growth defects are also observed upon depletion of the class- 2 myosin Myol $\mathrm{p}^{22}$. Thus, Myo2p-dependent changes in the morphology of mitochondria should be readily distinguishable from the defects in cell growth, actin cytoskeletal organization, and cytokinesis expected from the inhibition of other yeast myosins. Additionally, homology modeling of Myo2p in complex with the inhibitor predicts that PBP is a potent inhibitor of Myo2p function with six hydrogen bonds contributing to the interaction (Figure 3b). The interaction pattern between the protein matrix and the inhibitor is basically identical to $D d$ myosin-2 but most side chain replacements contribute to stronger binding. Replacement of $D d$ myosin-2 residues L431 and K265 with D421 and R255 in Myo2p leads to the formation of two additional hydrogen bonds and replacement of A424 with F414 increases the efficiency of the hydrophobic interaction between the corresponding part of the binding pocket and the inhibitor. The replacement of $D d$ myosin-2 residue $\mathrm{R} 428$ with an alanine residue leads to the loss of a van der Waals interaction but does not affect the local structure of the binding pocket. To facilitate the visual inspection of mitochondrial morphology, we used cells transfected with an expression vector for the production of mitochondria-targeted GFP ${ }^{23}$ (Fig. 4a). A yeast mutant strain with myo 2 expression under the control of the $\mathrm{TetO}_{7}$ promoter was 
used as control ${ }^{24}$. Repression of the $\mathrm{TetO}_{7}$ promoter by the addition of $10 \mu \mathrm{g} / \mathrm{ml}$ deoxycycline to the culture medium of this strain led to an apparent fragmentation of mitochondria (Fig. 4b). Similar phenotypic changes were observed when wild-type cells were exposed to $500 \mathrm{nM}$ PBP for 12 hours (Fig. 4c). At lower concentrations the phenotype was less severe and at 100 nM PBP the mitochondrial morphology closely resembled that of untreated wild-type cells. Yeast cells exposed to $500 \mathrm{nM}$ PBP showed normal cytokinesis and similar long-term growth kinetics as the wild type control cultures, indicating that inhibition is Myo2p specific.

\section{DISCUSSION}

Our results show that the halogenated pseudilin derivative PBP acts as allosteric inhibitor of myosin function. PBP binds in a pocket close to actin-binding residues that is open to the exterior and to the large cleft. Blebbistatin, the other structurally well characterized inhibitor of myosin motor activity, binds to a site $7.5 \AA$ away from the PBP pocket ${ }^{25}$. Blebbistatin is thought to inhibit $\mathrm{P}_{\mathrm{i}}$-release by sitting like a wedge in a hydrophobic pocket at the base of the large cleft. This prevents complete closure of the cleft and thereby stabilizes the ADP- $\mathrm{P}_{\mathrm{i}}$ transition state ${ }^{25}$. A similar stabilization of a product complex with low actin affinity has been observed with BTS, a specific inhibitor of fast skeletal muscle myosin- $2^{26}$. Transient kinetic performed with of $D d$ myosin-5b show that PBP affects both reaction steps leading to weak and strong actin binding states and indicate that other myosins are affected in a similar way by PBP. The addition of ATP to the IAM complex leads to the formation of the IAMT complex $\left(\mathbf{K}_{\mathbf{1 i}} \mathbf{k}_{\mathbf{C i}}\right)$, followed by dissociation of actin and formation of the IMT complex $\left(\mathbf{k}_{-6 \mathbf{i}}\right)$ (see Scheme Fig. 1h). These steps are 1.5 and 4 -fold slower in the presence of PBP. The reaction proceeds with the hydrolysis step $\left(k_{+2 \mathrm{i}}+k_{-2 \mathrm{i}}\right)$ leading to the formation of the ternary IMDP complex. The hydrolysis step shows the largest PBP-induced change with a 10 -fold reduction in the rate of the process. Hydrolysis is followed by rapid reassociation with actin leading to the formation of the IAMDP complex $\left(\mathbf{k}_{+7 \mathrm{i}}\right)$. The pentanary complex decomposes in the direction of phosphate release $\left(\mathbf{k}_{+3 \mathrm{i}}\right)$. Both $\mathrm{P}_{\mathrm{i}}$ release and the preceding step are not significantly affected by PBP. The cycle is closed with the formation of the IAM complex following the dissociation of ADP from the IAMD complex $\left(\mathbf{k}_{+4 i}\right)$. This step is the rate limiting step for the steady-state actin-activated ATPase activity of $D d$ myosin-5b in the presence of saturating concentrations of PBP. The PBP-induced changes in multiple rate constants lead to an increase of the actin-activated ATP-cycle time and shift the ratio of the time spend per cycle in strong and weak binding states. The slower movement of actin filaments in the in vitro motility assay observed with myosins from different classes and the myosin-2 dependent reduction in unloaded shortening velocity in single fiber experiments indicate that steps associated with ADP-release and ATP-induced dissociation of actomyosin are slowed down in the presence of $\mathrm{PBP}^{27}$. Additionally, the decrease in isometric tension generation observed in single fiber experiments indicates a PBP-induced redistribution in favor of cycle-time spend in non-force generating states, at least for skeletal muscle myosin-2 dependent processes. In the case of class- 5 myosins, which display weaker coupling between actin binding and ADP release, the addition of PBP leads to a similar extension of total cycle time but with a more dominant contribution of cycle-time spend in force generating states. In the case of $D d$ myosin-5b the coupling ratio $\left(\mathbf{K}_{4} / K_{4}\right)$ equals unity and becomes reversed in the presence of PBP $\left(K_{4 i} / K_{4 i}=0.08\right)$. As a consequence actin increases the affinity of $D d$ myosin- $5 \mathrm{~b}$ for $\mathrm{ADP}\left(\mathbf{K}_{4 \mathrm{i}}=1.23 \mu \mathrm{M}\right)$ and strong binding states become dominant under experimental conditions where ADP concentrations are not kept low.

Changes in the actin interface upon closure of the large cleft between U50 and L50 that are necessary for formation of the strongly bound myosin states on actin and efficient release of ATP hydrolysis products, involve large rearrangements of residues that are located near the PBP binding pocket ${ }^{28,29}$. The rearrangements are in turn coupled to significant movements of nucleotide-binding loop structures by a distortion of the central $\beta$-sheet. The switch-1 loop preceding $\beta 6$, the switch-2 loop following $\beta 5$, and the P-loop following $\beta 4$ undergo large conformational changes between the nucleotide-free and the catalytically competent structures. Cleft closure is thus coupled to the opening of the nucleotide binding pocket and 
the disruption of interactions that stabilize $\gamma$-phosphate binding, the coordination of the $\mathrm{Mg}^{2+}$ ion and, thereby, ADP binding ${ }^{30,31}$. Although the PBP-binding site is formed in part by residues that are implicated in these movements, the size and binding mode of PBP are not compatible with a mechanism were PBP interferes with cleft closure. Furthermore, the results of our kinetic, motility and tension measurements show conclusively that PBP does not block the formation of a strongly bound actomyosin complex and molecular modeling shows that the interactions of PBP with residues in the U50 are not affected by closure of the cleft. The effects of changes in the strut loop and loop-2 are more difficult to predict, however, even here cleft closure does not necessarily lead to a disruption of the interactions with PBP. This is supported by our experimental result. The observed changes in $\mathrm{IC}_{50}$ values for basal and actin activated ATPase activities indicate that the formation of a rigor-state actomyosin complex results in a less than 10-fold reduction in PBP binding affinity.

Our results using yeast as a model system to block a myosin-5 dependent process show that PBP has potential as a tool for cell motility studies. PBP readily permeates membranes and displays a clearly distinct selectivity for myosin isoforms. It is not a specific inhibitor of all myosins belonging to class- 5 but it strongly inhibits the function of individual members of class-5, such as vertebrate myosin-5a. An important aspect of the characterization of PBP is its role as lead compound for the development of more specific effectors of myosin function. The residues involved in PBP binding are only moderately conserved between the members of the different myosin classes, as reflected by the observed differences in the $\mathrm{IC}_{50}$ values for the myosin isoforms characterized in this study. Moreover, our results show that isoformspecific variations in the extent of PBP-mediated inhibition can be predicted at least in trend. The continuity of the PBP binding pocket with the $50 \mathrm{kDa}$ cleft indicates that the site can accommodate much bulkier compounds. As the affinity and specificity of drug-enzyme interactions tends to increase with their area of interaction, the structure-based design of compounds that extend into the cleft promises to improve their selectivity for individual myosin isoforms. Highly selective effectors of specific myosin isoforms have a wide range of potential therapeutic applications including cancer, heart failure and malaria ${ }^{32-34}$. Furthermore, the structural and functional framework provided by our results, can be used to design compounds, which upon binding induce specific functional changes in the myosin motor that are distinct from those observed with PBP.

\section{METHODS}

Protein preparation. We purified His-tagged motor domain constructs of myosin-1E, myosin-2, and myosin-5b from $D d$ comprising amino acids 1-698, 1-765, and 1-839

respectively, by $\mathrm{Ni}^{2+}$-chelate affinity chromatography ${ }^{35}$. Myosin-5a heavy meromyosin from chicken brain was provided by Dr. T. Scholz (Hannover Medical School, Germany). Rabbit fast skeletal muscle heavy meromyosin (HMM), F-actin, and pyrene-labeled F-actin were prepared as described ${ }^{36-38}$.

Synthesis of PBP. We synthesized PBP using a silver(I)-catalyzed cyclization to the pyrrole ring system ${ }^{39}$.

Steady-state kinetics. We measured basal and actin-activated $\mathrm{Mg}^{2+}$-ATPase activities using the NADH-coupled assay described previously ${ }^{40}$. PBP was added to the reaction mixture in the absence of nucleotide and incubated for 20 minutes before the reaction was started by the addition of ATP. Tests of the inhibitory potency of pseudilin and halogenated pseudilins were performed with HMM prepared from rabbit skeletal muscle myosin-2. Each reaction mixture including the controls contained 2.5\% DMSO that was used as solvent for the small organic compounds. Reactions containing HMM $(0.01 \mathrm{mg} / \mathrm{ml})$, assay buffer $(50 \mathrm{mM} \mathrm{KCl}, 5 \mathrm{mM}$ $\mathrm{CaCl}_{2}, 25 \mathrm{mM}$ Tris-HCl pH 7.5) and $25 \mu \mathrm{M}$ of the respective inhibitor were preincubated for $20 \mathrm{~min}$ at $37^{\circ} \mathrm{C}$ and the reaction was started by the addition of $50 \mu \mathrm{M}$ ATP. We terminated reactions after $20 \mathrm{~min}$ at $37^{\circ} \mathrm{C}$ by the addition of Biomol Green (Biomol, Hamburg, Germany). The amount of dye formed after 20 min color development at room temperature was determined by absorption measurements at $640 \mathrm{~nm}$ with a Tecan Infinite ${ }^{\mathrm{TM}}$ microplate 
reader (Crailsheim, Germany). Data were corrected for inhibitor absorption at 640nm and expressed as relative myosin ATPase activity (in \% of control).

Transient kinetics. We performed stopped-flow experiments with PiStar (Applied Photophysics, Leatherhead, U.K.) and Hi-Tech SF61 (TgK Scientific Limited, Bradford on Avon, U.K.) spectrophotometers using procedures and kinetic models described previously ${ }^{41,42}$. Tryptophan fluorescence was excited at $296 \mathrm{~nm}$ and detected after passing through a WG320 cut-off filter. Pyrene- and mant-fluorescence were excited at $365 \mathrm{~nm}$ and detected after passing through a KV $389 \mathrm{~nm}$ cut-off filter. Data analysis was performed with software provided by TgK Scientific Limited, Applied Photophysics and with Origin 7.0 (Originlab Corporation, Northampton, USA).

In vitro motility assay. We performed actin-sliding motility assays at $25^{\circ} \mathrm{C}$ using an Olympus IX81 Olympus, Hamburg, Germany) inverted fluorescence microscope as described previously ${ }^{43}$. Equine cytochrome $\mathrm{C}$ was used as blocking agent instead of bovine serum albumin. Average sliding velocities were determined from the Gaussian distribution of automatically tracked actin filaments using DiaTrack 3.0 (Semasopht, Chavannes, Switzerland). Additional data analysis was performed with Origin 7.0.

Crystallization and data collection. We grew crystals of motor domain construct M761$\mathrm{c} 14^{43}$ in the presence of sodium meta-vanadate and ADP or sodium meta-vanadate, ADP, and PBP at $4{ }^{\circ} \mathrm{C}$ using the hanging drop geometry. $2 \mu \mathrm{L}$ of complex solution and $2 \mu \mathrm{L}$ of reservoir solution were mixed, the latter contained $50 \mathrm{mM}$ HEPES pH 7.4, $140 \mathrm{mM} \mathrm{NaCl}, 11 \% \mathrm{w} / \mathrm{v}$ PEG8000, 2\% (v/v) MPD, $5 \mathrm{mM} \mathrm{MgCl}_{2}, 5 \mathrm{mM}$ DTT, and $1 \mathrm{mM} \mathrm{EGTA.} \mathrm{Prior} \mathrm{to} \mathrm{diffraction}$ data collection, we soaked crystals for 5 minutes at $4{ }^{\circ} \mathrm{C}$ in a cryo-protection solution containing reservoir solution supplemented with $25 \%$ ethylene glycol, $2 \mathrm{mM}$ sodium metavanadate, $2 \mathrm{mM}$ ADP. Subsequently, crystals were flash-cooled in liquid nitrogen. We collected diffraction data for the myosin-2 motor domain $\mathrm{Mg}^{2+} \mathrm{ADP}-m e t a$-vanadate complex at EMBL beamline X11 at the DORIS storage ring, DESY, Hamburg. Diffraction data for the complex with PBP were collected in house using an X8PROTEUM system equipped with cryo-system, $\kappa$-goniometer and CCD-detector and processed with PROTEUM2 software (Bruker AXS, Karlsruhe, Germany). We solved the structures using $\mathrm{C} 222_{1}$ data by molecular replacement using 1VOM as initial model (Table 2).

Single fiber mechanics. We isolated single fibers from bundles of chemically skinned fibers of the M. psoas of rabbits, as described previously ${ }^{44,45}$. One end of the isolated fibers was attached to the lever of a modified moving coil galvanometer for length control, the other to a strain gauge force transducer (Akers, Horten, Norway). Overall fiber length was set such that sarcomere length was $2.4 \mu \mathrm{m}$. We measured sarcomere length by diffraction of a $633 \mathrm{~nm}$ helium-neon laser beam. Composition of relaxing and activating solutions were identical to our previous work ${ }^{46,47}$. Ionic strength of activating and pre-activating solutions was adjusted to $170 \mathrm{mM}$ and $50 \mathrm{mM}$ for the solution to measure stiffness of relaxed fibers at 'low ionic strength'. Ionic strength was adjusted by addition of appropriate amounts of potassium propionate. Experimental temperature was set to $5{ }^{\circ} \mathrm{C}$. Unloaded shortening was measured by slack-test ${ }^{48}$.

Molecular modeling. We used the motor domain structures of $D d$ myosin-1E (PDB entry $1 \mathrm{LKX}$ ) and chicken myosin-5a (PDB entry 1OE9) to model the complexes of class-1 and class-5 myosins with PBP. Initial models were created by inserting PBP coordinates from the superimposed myosin-2(PBP) complex. Next molecular mechanics energy minimization procedures were performed for PBP in the new protein environment using the CNS software suit $^{49}$. The resulting structures were subjected to a more precise quantum chemical energy minimization procedure, using ab initio DFT QM/MM calculations with the $6-31 \mathrm{G}^{*}$ basis set of atomic orbitals and B3LYP hybrid functional. The quantum chemical calculations were 
performed using the PC GAMESS version developed by Alex A. Granovsky (http://www.classic.chem.msu.su/gran/gamess/index.html) of the GAMESS (US) Quantum Chemistry package ${ }^{50}$.

Myo2p-dependent changes in mitochondrial morphology. Yeast strains producing mitochondria-targeted GFP were supplied by Dr. B. Westermann ${ }^{23}$. Repression of the $\mathrm{TetO}_{7}$ promoter that controls myo2 in one of the mutant strains was achieved by the addition of 10 $\mu \mathrm{g} / \mathrm{mL}$ deoxycycline to the culture medium ${ }^{24}$. Cells were analyzed at the end of the logarithmic growth phase. Images were acquired using a LSM 510 confocal microscope (Carl Zeiss AG, Jena, Germany) using a 63x oil immersion objective and an ORCA camera (Hamamatsu Photonics GmbH, Herrsching, Germany) for bright-field imaging.

Accession codes. Protein Data Bank (PDB): Coordinates for the myosin-2 motor domain $\mathrm{ADP} \cdot \mathrm{VO}_{3}$ and myosin-2 motor domain $-\mathrm{ADP} \cdot \mathrm{VO}_{3}-\mathrm{PBP}$ complexes have been deposited with the accession codes $2 \mathrm{JJ} 9$ and $2 \mathrm{JHR}$, respectively.

Note: Supplementary information is available on the Nature Structural \& Molecular Biology website.

\section{ACKNOWLEDGEMENTS}

We thank S. Fujita-Becker (Max-Planck-Institute for Medical Research, Heidelberg), B. Westermann (University of Bayreuth), T. Scholz, A. Schweda (Hannover Medical School) A. Malnasi-Csizmadia and M. Kovacs (Eötvös Lorand University, Budapest), and C.R. Bagshaw (University of Leicester) for reagents; M.A. Geeves (University of Kent, Canterbury) for discussions; C. Thiel, C. Wassmann, Y. Henker, and L. Litz for excellent technical assistance. We are grateful to the staff of beamline EMBL X11 at the DORIS storage ring of DESY for their support.

\section{COMPETING INTERESTS STATEMENT}

The authors declare that they have no competing financial interests.

\section{AUTHOR CONTRIBUTIONS}

R.F. performed crystallization, structure determination, and molecular modeling; M.B. performed the initial kinetic screening of the pseudilin derivatives and the yeast assays; G.T. supervised and co-designed the protein preparation, kinetic and in vitro motility assays, and performed the transient kinetic analysis with F.K.H. and M.H.T.; F.K.H performed in vitro motility assays and steady-state analysis; M.H.T. performed transient kinetic experiments; P.B. crystallized the protein inhibitor complexes; B.B. designed and performed single fiber experiments; R.M. synthesized PBP; H.-J.K. designed and supervised compound synthesis; H.O.G. designed and supervised the initial kinetic screening of PBP and the yeast assays; D.J.M. supervised kinetic analysis, crystallization and data collection, molecular modeling, in vitro motility analysis, advised on the design of the experiments, and wrote the manuscript with G.T., R.F., H.O.G., B.B., and H.-J.K. 
Figure 1 Inhibition of myosin functions by PBP. (a) Effect of PBP on the steady state actin-activated ATPase activity of $D d$ myosin-5b. Actin-activated ATPase activities in the absence ( $\boldsymbol{a})$ and presence of $25 \mu \mathrm{M}$ PBP $(\boldsymbol{\nabla})$ are shown. (b) Concentrationdependence of the inhibition of the actin-activated ATPase activity displayed by different myosin isoforms in the presence of 0 to $150 \mu \mathrm{M}$ PBP. The semi-logarithmic plot shows the concentration dependence for myosin-1E (o), myosin-2 ( $\mathbf{\square}), D d$ myosin-5b $(\Delta)$, and chicken myosin-5a $(\boldsymbol{\nabla})$. The concentrations of PBP required for half-maximal inhibition $\left(\mathrm{IC}_{50}\right)$ of the different myosin motors were determined from sigmoidal fits of the data. All parameters are summarized in Table 1. (c) Fluorescence transients obtained upon mixing $1 \mu \mathrm{M} D d$ myosin-5b with $10 \mu \mathrm{M}$ mantATP in the absence and presence of $5 \mu \mathrm{M}, 25 \mu \mathrm{M}, 50 \mu \mathrm{M}$ and $100 \mu \mathrm{M}$ PBP. The concentration dependence of the amplitude of the fast phase is shown in the inset.

(d) Rate constants for ATP binding and hydrolysis were determined by following the time-dependent changes in the intrinsic protein fluorescence of $D d$ myosin- $5 \mathrm{~b}$. Addition of $25 \mu \mathrm{M}$ PBP leads to a 14-fold reduction in the apparent second-order rate constant for ATP binding and a 10-fold reduction of the rate of ATP hydrolysis. (Supplemental Methods online). (e) PBP-mediated inhibition of chicken myosin-5a in the in vitro motility assay. The histograms show the average sliding velocity of rhodamine-phalloidin-labeled actin filaments in the absence and presence of $10 \mu \mathrm{M}$ PBP. Chicken myosin-5a moves actin filaments with an average velocity of $0.6 \pm 0.05$ $\mu \mathrm{m} \mathrm{s}^{-1}$ (dark grey bars); addition of $10 \mu \mathrm{M}$ PBP into the same flow cell decreases the average sliding velocity to $0.013 \pm 0.002 \mu \mathrm{m} \mathrm{s}^{-1}$ (light grey bars). (f) Inhibition of steady-state level of isometric tension generated by skinned fibers of rabbit psoas muscle. Single fibers were first pre-incubated for 90 min with $200 \mathrm{mM}$ PBP in relaxing solution before transfer to the activating solution containing the same PBP concentration. (g) Inhibition of unloaded shortening velocity by PBP. Each symbol represents the amplitude of imposed slack (length change) vs. time required for taking up the imposed slack by active shortening ${ }^{48}$. The slopes of the black curves give the velocity of unloaded shortening at maximum ( $\boldsymbol{\square})$ and half-maximum ( $\boldsymbol{\Delta}$ ) $\mathrm{Ca}^{2+}$-activation in the absence of PBP. Changes in the slopes indicate that shortening velocity declines as shortening progresses in the absence of the inhibitor. The dotted lines show unloaded shortening after the fiber was incubated for 2 hours in relaxing solution containing $200 \mu \mathrm{M}$ PBP $(\square)$ and following wash out of PBP for 15 $(\mathrm{O})$ and 20 minutes $(\Delta)$. (h) Scheme describing the mechanism for PBP inhibition of basal and actin-activated myosin ATPase activity. The symbols used are as follows: A, actin; D, ADP; I, PBP; M, myosin; P, phosphate; T, ATP. Rate and equilibrium constants in the absence and presence of actin are distinguished by the use of italics versus bold type. Equilibrium constants are expressed as proceeding from left to right/top to bottom in this scheme. Error bars indicate s.d.

Figure 2 Structure of the myosin-2 motor domain in complex with PBP and $\mathrm{Mg}^{2+}$ ADP-meta-vanadate. (a) Sections of the $2 \mathrm{~F}_{\mathrm{o}}-\mathrm{F}_{\mathrm{c}}$ electron density omit map showing the $\mathrm{Mg}^{2+} \mathrm{ADP}-$ meta-vanadate binding site and (b) the PBP binding site. The map was calculated using the final model of the myosin-2 motor domain with inhibitor and substrate omitted. The map was contoured at $1.0 \mathrm{\sigma}$. (c) Overall view of myosin motor domain in ribbon representation. PBP is shown in spheres mode and nucleotide in stick representation. Yellow, 25-kDa domain; red, upper 50-kDa domain; grey, lower 50-kDa domain, blue, 20-kDa domain. FHC loop, strut loop, loop 2 and the connecting helix-loop-helix motif are shown in light brown. (d) Close-up view of the PBP binding site. Selected residues interacting with PBP are in stick representation. Coloring as in (c).

Figure 3 PBP binding site and contact residue conservation for different myosin isoforms. Myosin residues interacting with PBP are shown as predicted by molecular modeling for (a) Dd myosin-1E; (b) Sc Myo2p; (c) Gg myosin-5a; (d) Dd myosin-5b. 
Figure 4 Sc Myo2p-dependent changes in mitochondrial morphology. In yeast cells, the class-5 myosins Myo2 $p$ is responsible for the distribution of mitochondria between mother and daughter cells during cytokinesis and has been shown to affect mitochondrial morphology during interphase. (a) Wild-type yeast cells; (b) Myo2pdepleted cells; (c) the phenotype of Myo2p-depleted cells is phenocopied by exposure of wild-type cells to 500 nM PBP. Insets show the same cells viewed under transmitted light. Bar: $5 \mu \mathrm{m}$.

\section{References}

1. Sokac, A.M., Schietroma, C., Gundersen, C.B. \& Bement, W.M. Myosin-1c couples assembling actin to membranes to drive compensatory endocytosis. Dev. Cell 11, 629-640 (2006).

2. Arden, S.D., Puri, C., Au, J.S., Kendrick-Jones, J. \& Buss, F. Myosin VI is required for targeted membrane transport during cytokinesis. Mol. Biol. Cell 18, 4750-4761 (2007).

3. Zhang, H. et al. Myosin-X provides a motor-based link between integrins and the cytoskeleton. Nat. Cell Biol. 6, 523-531 (2004).

4. Cavellan, E., Asp, P., Percipalle, P. \& Farrants, A.K. The WSTF-SNF2h chromatin remodeling complex interacts with several nuclear proteins in transcription. J. Biol. Chem. 281, 16264-16271 (2006).

5. van den Boom, F., Dussmann, H., Uhlenbrock, K., Abouhamed, M. \& Bahler, M. The Myosin IXb motor activity targets the myosin IXb RhoGAP domain as cargo to sites of actin polymerization. Mol. Biol. Cell 18, 1507-1518 (2007).

6. Weber, K.L., Sokac, A.M., Berg, J.S., Cheney, R.E. \& Bement, W.M. A microtubule-binding myosin required for nuclear anchoring and spindle assembly. Nature 431, 325-329 (2004).

7. Ohri, R.V. et al. A Re(V)-catalyzed $\mathrm{C}-\mathrm{N}$ bond-forming route to human lipoxygenase inhibitors. Org. Lett. 7, 2501-2504 (2005).

8. Burkholder, P.R., Pfister, R.M. \& Leitz, F.H. Production of a pyrrole antibiotic by a marine bacterium. Appl. Microbiol. 14, 649-53 (1966).

9. Taft, M.H. et al. Dictyostelium myosin-5b is a conditional processive motor. J. Biol. Chem. 283 , 26902-26910 (2008).

10. Hiratsuka, T. Fluorescence properties of 2' (or 3')-O-(2,4,6-trinitrophenyl) adenosine 5'-triphosphate and its use in the study of binding to heavy meromyosin ATPase. Biochim. Biophys. Acta 453, 293297 (1976).

11. Webb, M.R. A continuous spectrophotometric assay for inorganic phosphate and for measuring phosphate release kinetics in biological systems. Proc. Natl. Acad. Sci. USA 89, 4884-4887(1992).

12. Malnasi-Csizmadia, A., Kovacs, M., Woolley, R.J., Botchway, S.W. \& Bagshaw, C.R. The dynamics of the relay loop tryptophan residue in the Dictyostelium myosin motor domain and the origin of spectroscopic signals. J. Biol. Chem. 276, 19483-19490 (2001).

13. Geeves, M.A., Fedorov, R. \& Manstein, D.J. Molecular mechanism of actomyosin-based motility. Cell. Mol. Life Sci. 62, 1462-1477 (2005).

14. Brenner, B., Yu, L.C., Greene, L.E., Eisenberg, E. \& Schoenberg, M. Ca ${ }^{2+}$-sensitive cross-bridge dissociation in the presence of magnesium pyrophosphate in skinned rabbit psoas fibers. Biophys. J. 50, 1101-1108 (1986).

15. Dominguez, R., Freyzon, Y., Trybus, K.M. \& Cohen, C. Crystal structure of a vertebrate smooth muscle myosin motor domain and its complex with the essential light chain: visualization of the prepower stroke state. Cell 94, 559-571 (1998).

16. Sasaki, N., Ohkura, R. \& Sutoh, K. Insertion or deletion of a single residue in the strut sequence of Dictyostelium myosin II abolishes strong binding to actin. J. Biol. Chem. 275, 38705-38709 (2000).

17. Boldogh, I.R., Ramcharan, S.L., Yang, H.C. \& Pon, L.A. A type V myosin (Myo2p) and a Rab-like G-protein (Ypt11p) are required for retention of newly inherited mitochondria in yeast cells during cell division. Mol. Biol. Cell 15, 3994-4002 (2004).

18. Itoh, T., Watabe, A., Toh, E.A. \& Matsui, Y. Complex formation with Ypt11p, a Rab-type small GTPase, is essential to facilitate the function of Myo2p, a class V myosin, in mitochondrial distribution in Saccharomyces cerevisiae. Mol. Cell Biol. 22, 7744-7757 (2002).

19. Shepard, K.A. et al. Widespread cytoplasmic mRNA transport in yeast: identification of 22 budlocalized transcripts using DNA microarray analysis. Proc. Natl. Acad. Sci. USA 100, 11429-34 (2003).

20. Simon, V.R., Swayne, T.C. \& Pon, L.A. Actin-dependent mitochondrial motility in mitotic yeast and cell-free systems: identification of a motor activity on the mitochondrial surface. J. Cell Biol. 130, 345-354 (1995).

21. Goodson, H.V., Anderson, B.L., Warrick, H.M., Pon, L.A. \& Spudich, J.A. Synthetic lethality screen identifies a novel yeast myosin I gene (MYO5): myosin I proteins are required for polarization of the actin cytoskeleton. J. Cell Biol. 133, 1277-1291 (1996).

22. Lord, M., Laves, E. \& Pollard, T.D. Cytokinesis depends on the motor domains of myosin-II in fission yeast but not in budding yeast. Mol. Biol. Cell 16, 5346-5355 (2005). 
23. Westermann, B. \& Neupert, W. Mitochondria-targeted green fluorescent proteins: convenient tools for the study of organelle biogenesis in Saccharomyces cerevisiae. Yeast 16, 1421-1427 (2000).

24. Mnaimneh, S. et al. Exploration of essential gene functions via titratable promoter alleles. Cell 118, 31-44 (2004).

25. Allingham, J.S., Smith, R. \& Rayment, I. The structural basis of blebbistatin inhibition and specificity for myosin II. Nat. Struct. Mol. Biol. 12, 378-379 (2005).

26. Cheung, A. et al. A small-molecule inhibitor of skeletal muscle myosin II. Nat. Cell Biol. 4, 83-88 (2002).

27. Nyitrai, M. et al. What limits the velocity of fast-skeletal muscle contraction in mammals? J. Mol. Biol. 355, 432-442 (2006).

28. Reubold, T.F., Eschenburg, S., Becker, A., Kull, F.J. \& Manstein, D.J. A structural model for actininduced nucleotide release in myosin. Nat. Struct. Biol. 10, 826-830 (2003).

29. Coureux, P.D. et al. A structural state of the myosin $\vee$ motor without bound nucleotide. Nature $\mathbf{4 2 5}$, 419-423 (2003).

30. Reubold, T.F., Eschenburg, S., Becker, A., Kull, F.J. \& Manstein, D.J. A structural model for actininduced nucleotide release in myosin. Nat. Struct. Biol. 10, 826-830 (2003).

31. Coureux, P.D. et al. A structural state of the myosin $V$ motor without bound nucleotide. Nature $\mathbf{4 2 5}$, 419-423 (2003).

32. Dunn, T.A. et al. A novel role of myosin VI in human prostate cancer. Am. J. Pathol. 169, 18431854 (2006).

33. Tavares, M., Rezlan, E., Vostroknoutova, I., Khouadja, H. \& Mebazaa, A. New pharmacologic therapies for acute heart failure. Crit. Care. Med. 36, S112-120 (2008).

34. Green, J.L. et al. The MTIP-myosin A complex in blood stage malaria parasites. J. Mol. Biol. 355, 933-941 (2006).

35. Manstein, D.J. \& Hunt, D.M. Overexpression of myosin motor domains in Dictyostelium: screening of transformants and purification of the affinity tagged protein. J. Mus. Res. Cell Motil. 16, 325-332 (1995).

36. Margossian, S.S. \& Lowey, S. Preparation of myosin and its subfragments from rabbit skeletal muscle. Meth. Enzymol. 85 Pt B, 55-71 (1982).

37. Lehrer, S.S. \& Kerwar, G. Intrinsic fluorescence of actin. Biochemistry 11, 1211-1217 (1972).

38. Criddle, A.H., Geeves, M.A. \& Jeffries, T. The use of actin labelled with N-(1-pyrenyl)iodoacetamide to study the interaction of actin with myosin subfragments and troponin/tropomyosin. Biochem. J. 232, 343-349 (1985).

39. Agarwal, S. \& Knölker, H.-J. A novel pyrrole synthesis. Org. Biomol. Chem. 2, 3060-3062 (2004).

40. Furch, M., Geeves, M.A. \& Manstein, D.J. Modulation of actin affinity and actomyosin adenosine triphosphatase by charge changes in the myosin motor domain. Biochemistry 37, 6317-6326 (1998).

41. Batra, R., Geeves, M.A. \& Manstein, D.J. Kinetic analysis of Dictyostelium discoideum myosin motor domains with glycine-to-alanine mutations in the reactive thiol region. Biochemistry 38, 61266134 (1999).

42. Fujita-Becker, $\mathrm{S}$. et al. Changes in $\mathrm{Mg}^{2+}$ ion concentration and heavy chain phosphorylation regulate the motor activity of a class I myosin. J. Biol. Chem. 280, 6064-6071 (2005).

43. Fedorov, R., Witte, G., Urbanke, C., Manstein, D.J. \& Curth, U. 3D structure of Thermus aquaticus single-stranded DNA-binding protein gives insight into the functioning of SSB proteins. Nucleic Acids Res. 34, 6708-6717 (2006).

44. Brenner, B. Technique for stabilizing the striation pattern in maximally calcium-activated skinned rabbit psoas fibers. Biophys. J. 41, 99-102 (1983).

45. Kraft, T. et al. Equilibration and exchange of fluorescently labeled molecules in skinned skeletal muscle fibers visualized by confocal microscopy. Biophys. J. 69, 1246-1258 (1995).

46. Brenner, B. \& Eisenberg, E. Rate of force generation in muscle: correlation with actomyosin ATPase activity in solution. Proc. Natl. Acad. Sci. USA 83, 3542-3546 (1986).

47. Brenner, B. Effect of $\mathrm{Ca}^{2+}$ on cross-bridge turnover kinetics in skinned single rabbit psoas fibers: implications for regulation of muscle contraction. Proc. Natl. Acad. Sci. USA 85, 3265-3269 (1988).

48. Edman, K.A. The velocity of unloaded shortening and its relation to sarcomere length and isometric force in vertebrate muscle fibres. J. Physiol .291, 143-159 (1979).

49. Brunger, A.T. et al. Crystallography \& NMR system: A new software suite for macromolecular structure determination. Acta Crystallographica Section D-Biol. Crystallog. 54, 905-921 (1998).

50. Schmidt, M.W. et al. The General Atomic and Molecular Electronic Structure System. J. Comput. Chem. 14, 1347-1363 (1993). 
Table 1. Kinetic analysis of PBP inhibition

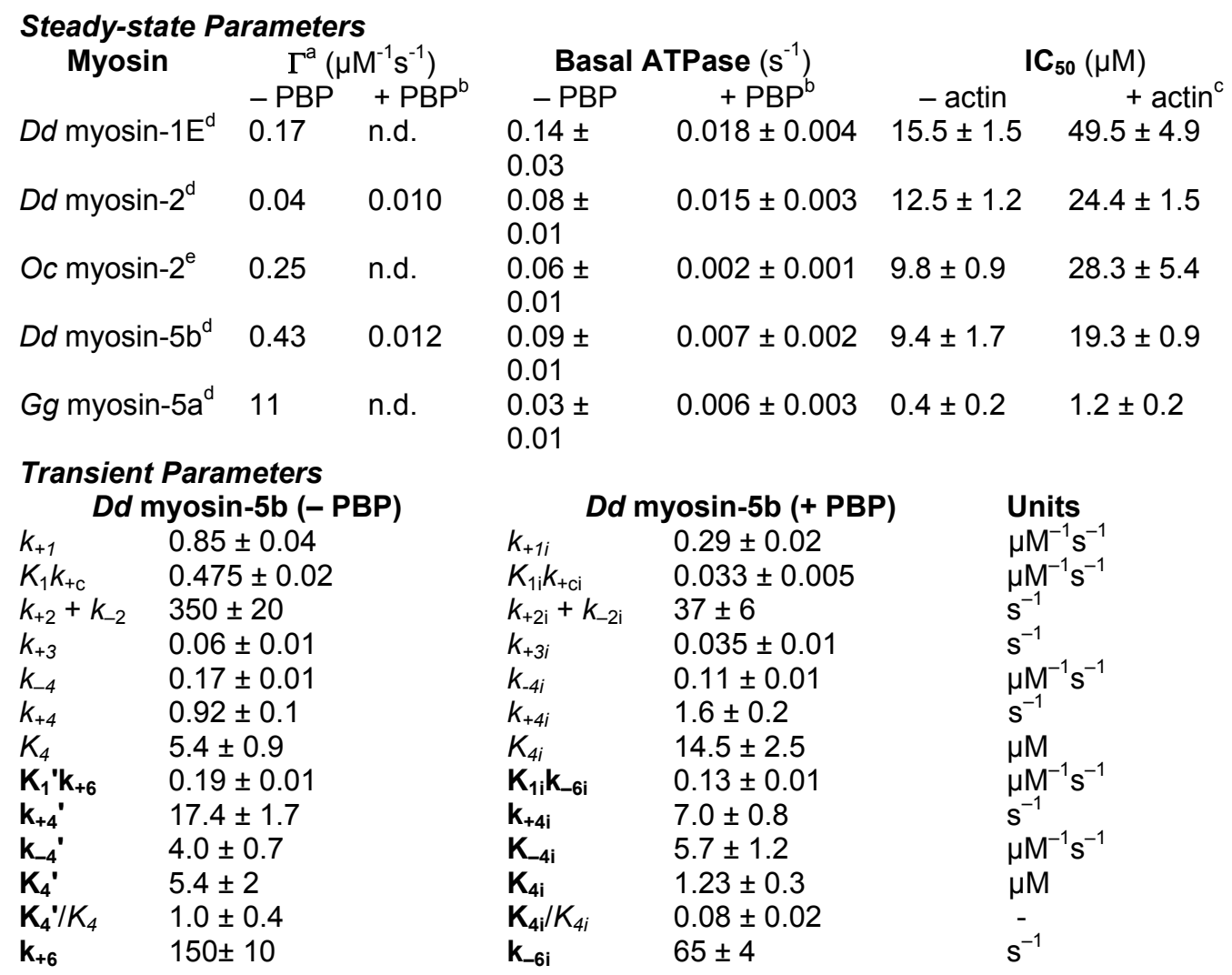

${ }^{a} \Gamma$ corresponds to the apparent second order rate constant for actin binding $\left(k_{\text {cat }} / K_{\text {app }}\right)$ that can be accurately determined from the initial slope of a plot $k_{\mathrm{obs}}$ against actin concentration at F-actin concentrations much smaller than $K_{\text {app }} ;{ }^{b}$ measured in the presence of $25 \mu \mathrm{M} \mathrm{PBP} ;{ }^{c}$ $30 \mu \mathrm{M}$ actin was added; 'single headed motor domain construct; ${ }^{\mathrm{e}}$ heavy meromyosin fragment. Abbreviations: n.d., not determined; $D d$, D. discoideum; $G g$, Gallus gallus; Oc, Oryctolagus cuniculus. 
Table 2 Data collection and refinement statistics

\begin{tabular}{|c|c|c|}
\hline & Myosin-2 - ADP.VO ${ }_{3}-\mathrm{PBP}$ & Myosin-2 - ADP.VO \\
\hline \multicolumn{3}{|l|}{ Data collection } \\
\hline Space group & $C 222_{1}$ & $C 222_{1}$ \\
\hline \multicolumn{3}{|l|}{ Cell dimensions } \\
\hline$a, b, c(\AA)$ & $89.8,150.5,154.6$ & $89.4,147.2,153.8$ \\
\hline$\alpha, \beta, \gamma\left({ }^{\circ}\right)$ & $90,90,90$ & $90,90,90$ \\
\hline Resolution $(\AA)$ & $20.0-2.8$ & $20.0-2.1$ \\
\hline$R_{\text {sym }}(\%)$ & $8.8(40.4)^{*}$ & $5.8(40.8)$ \\
\hline$I / \sigma \mid$ & $10.9(2.4)$ & $24.4(5.0)$ \\
\hline Completeness (\%) & $98.2(95.5)$ & $98.2(98.5)$ \\
\hline Redundancy & $5.2(4.0)$ & $9.5(6.9)$ \\
\hline \multicolumn{3}{|l|}{ Refinement } \\
\hline Resolution $(\AA)$ & $8.0-2.8$ & $8.0-2.3$ \\
\hline$R_{\text {work }} / R_{\text {free }}(\%)$ & $21.7 / 26.5$ & $22.5 / 27.5$ \\
\hline $\begin{array}{l}\text { No. reflections } \\
\text { working/test set }\end{array}$ & $22214 / 1178$ & $43111 / 2268$ \\
\hline \multicolumn{3}{|l|}{ No. atoms } \\
\hline Protein & 6240 & 5535 \\
\hline Ligands/ions & $48 / 1$ & $31 / 1$ \\
\hline Water & 477 & 508 \\
\hline \multicolumn{3}{|l|}{$B$-factors $\left(\AA^{2}\right)$} \\
\hline Protein & 41.9 & 42.7 \\
\hline Ligands/ions & $31.3 / 31.2$ & $30.8 / 28.9$ \\
\hline Water & 39.2 & 51.5 \\
\hline \multicolumn{3}{|l|}{ R.m.s. deviations } \\
\hline Bond lengths $(\AA)$ & 0.009 & 0.006 \\
\hline Bond angles $\left(^{\circ}\right)$ & 1.4 & 1.3 \\
\hline
\end{tabular}


a
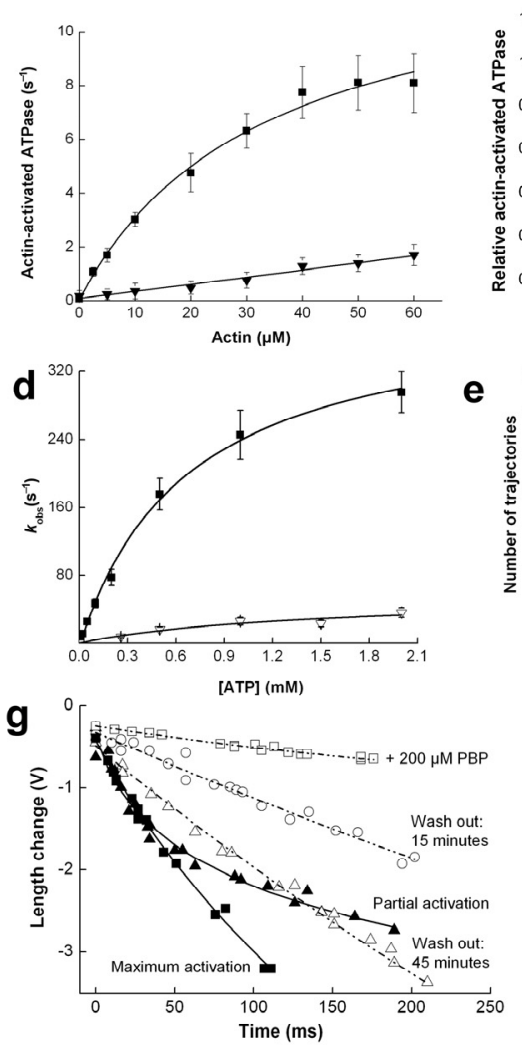

b

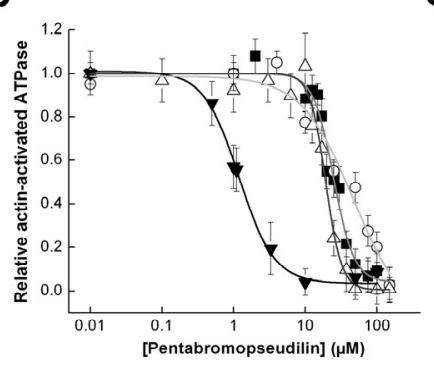

e

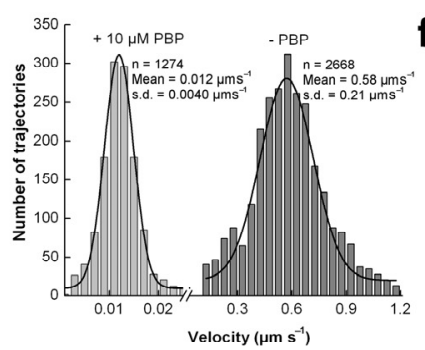

C

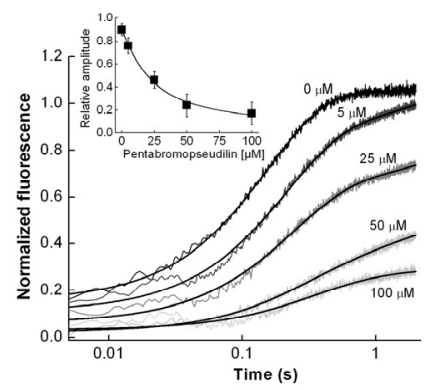

h $\quad \mathrm{M} \stackrel{\kappa_{1} K_{0}}{\rightleftharpoons} \mathrm{MT} \stackrel{K_{2}}{\rightleftharpoons} \mathrm{MDP} \stackrel{\kappa_{3}}{\rightleftharpoons} \mathrm{MD} \stackrel{K_{4}}{\rightleftharpoons} \mathrm{M}$

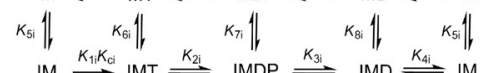

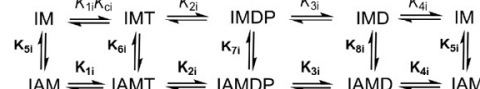

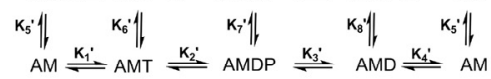

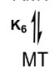

Figure 1 

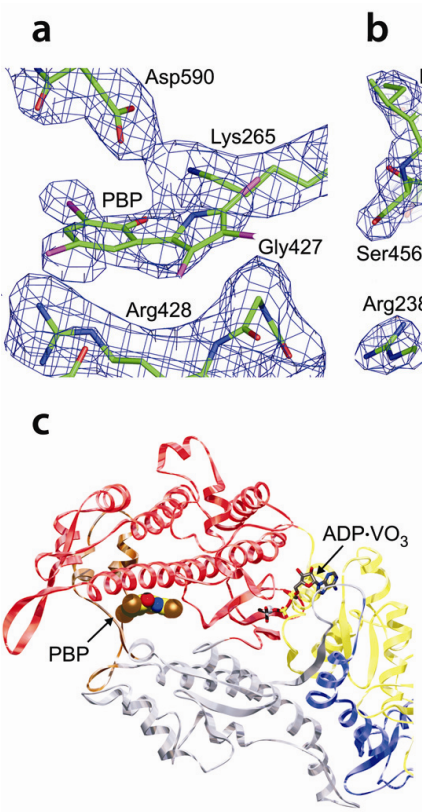

b

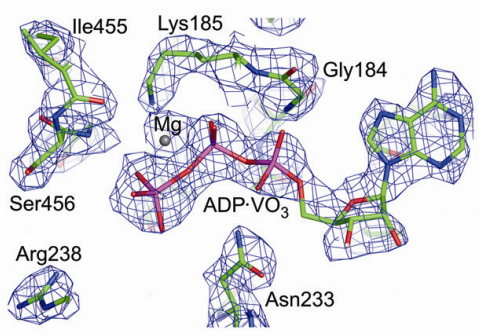

d

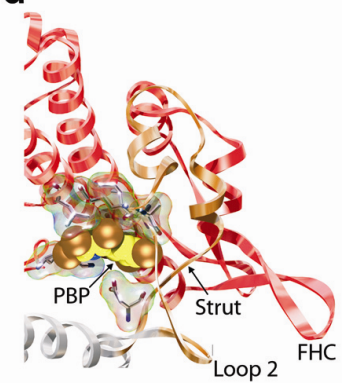

Figure 2 

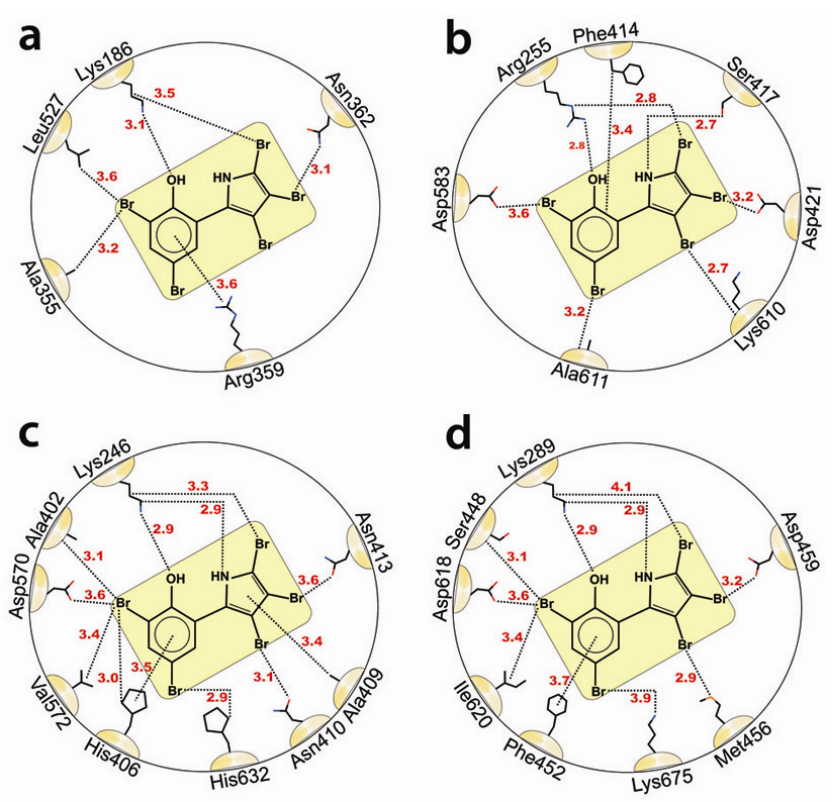

Figure 3 


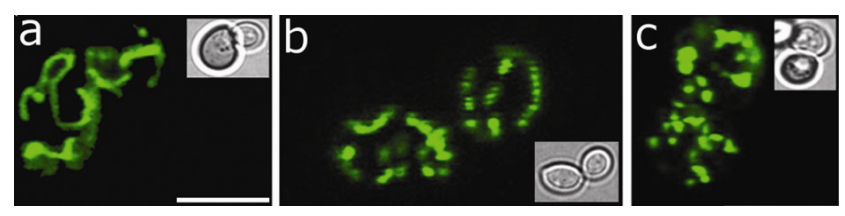

Figure 4 


\section{ONLINE SUPPLEMENTARY DATA}

\section{The mechanism of pentabromopseudilin inhibition of myosin motor activity}

Roman Fedorov $^{1}$, Markus Böhl ${ }^{2}$, Georgios Tsiavaliaris ${ }^{3}$, Falk K. Hartmann ${ }^{3}$, Manuel H. Taft ${ }^{3}$, Petra Baruch ${ }^{1}$, Bernhard Brenner ${ }^{4}$, René Martin ${ }^{5}$, Hans-Joachim Knölker ${ }^{5}$, Herwig O. Gutzeit ${ }^{2} \&$ Dietmar J. Manstein ${ }^{1,3^{*}}$

${ }^{1}$ Research Centre for Structure Analysis, OE8830, Hannover Medical School, 30623 Hannover, Germany; ${ }^{2}$ Department of Zoology, TU Dresden, Zellescher Weg 20b, 01217 Dresden; ${ }^{3}$ Institute for Biophysical Chemistry, OE4350, Hannover Medical School, 30623 Hannover, Germany; ${ }^{4}$ Institute for Molecular and Cellular Physiology, OE4210, Hannover Medical School, 30623 Hannover, Germany; ${ }^{5}$ Department of Chemistry, TU Dresden, Bergstrasse 66, 01069 Dresden, Germany

*Correspondence should be addressed to DJM (manstein@bpc.mh-hannover.de).

Supplementary Data include: $\quad$ Supplementary Figures 1, 2, 3 and 4

Supplementary Methods

Supplementary References 

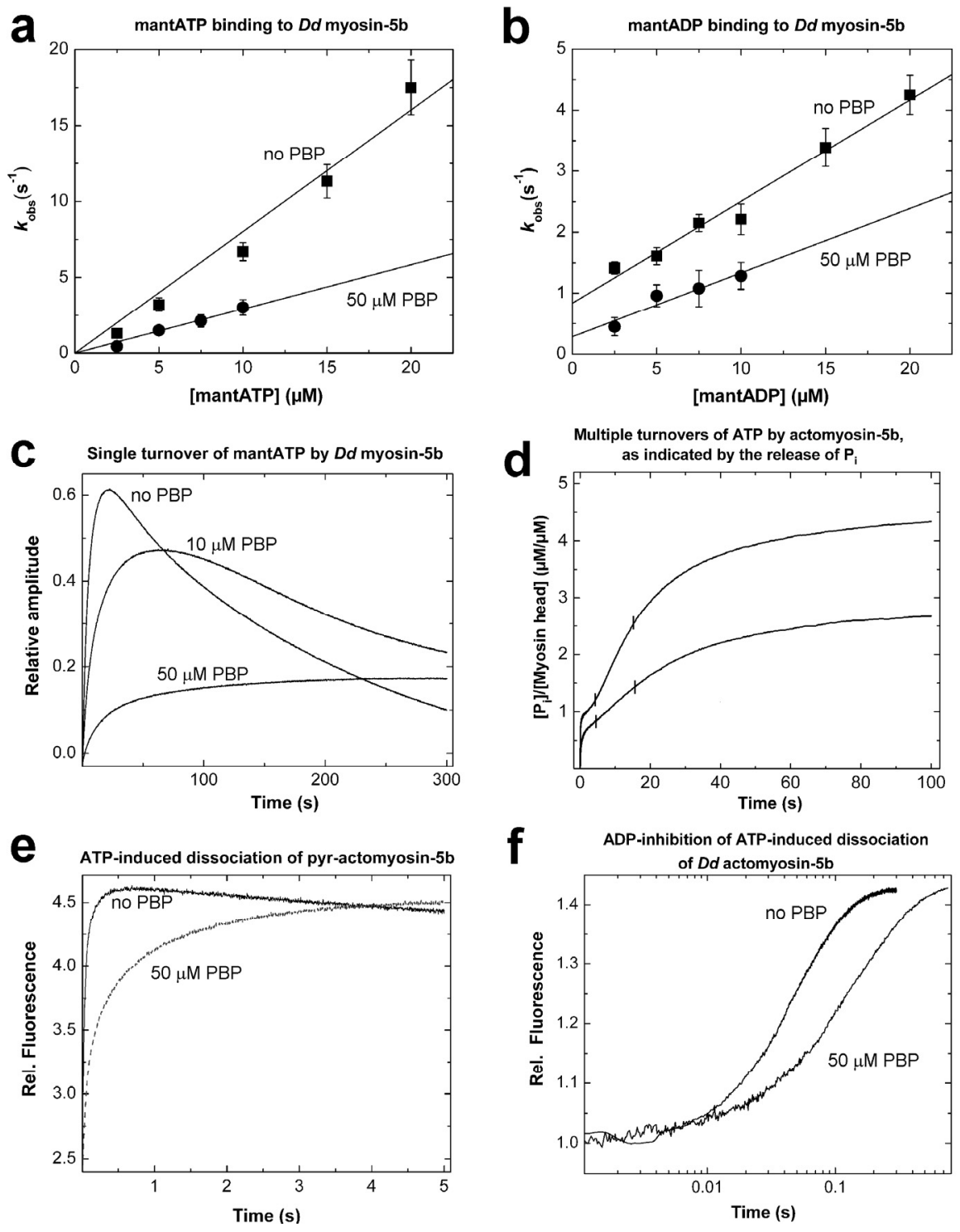

Supplemental Figure 1 Transient kinetic analysis. (a) MantATP binding to the $D d$ myosin-5b motor domain in the absence ( $\bullet$ ) and presence $(\bullet)$ of $50 \mu \mathrm{M}$ PBP. The apparent second order rate constant for mantATP binding was determined from the slope of the straight line fitted to the data. (b) MantADP binding to $D d$ myosin-5b in the absence $(\bullet)$ and presence $(\bullet)$ of $50 \mu \mathrm{M}$ PBP. The observed rate constants were determined by fitting the time courses of the fluorescence change to single exponentials and plotted against the mantADP concentration. (c) Single turnover experiments showing the changes in fluorescence signal upon mixing $0.5 \mu \mathrm{M} D d$ myosin- $5 \mathrm{~b}$ with $0.4 \mu \mathrm{M}$ mantATP in the presence of 0,10 and $50 \mu \mathrm{M}$ PBP over a time range of 300 seconds. In the absence of PBP the time evolution of the reaction can be separated in three phases: an initial rise in mant-fluorescence corresponding to binding of mantATP, a short plateau phase determined by the rate of the conformational change that follows mantATP binding, and a drop in mant- 
fluorescence corresponding to release and rebinding of mantADP $\left(k_{-4}+k_{+4}\right)$. The addition of $10 \mu \mathrm{M}$ PBP leads to a $20 \%$ loss of the amplitude reached at the plateau stage, a 2.5-fold reduction in the observed rate for the initial phase from 0.093 to $0.037 \mathrm{~s}^{-1}$, the formation of a more extended plateau phase, and a slight decrease in the observed rate constant for the third phase from 0.054 to $0.034 \mathrm{~s}^{-1}$. Addition of saturating concentrations of PBP leads to a 7-fold reduction in the observed rate for the initial phase. The amplitude reached at the plateau stage is more than $60 \%$ reduced and the plateau extents into the 1,000 second range. (d) Multiple-turnover of ATP by $D d$ myosin-5b in the absence and presence of $50 \mu \mathrm{M}$ PBP. The transients show the time evolution of the release of $P_{i}$, as measured with a linked enzyme assay. The observed three phases are indicated by vertical lines. The amplitudes and rate constants of the burst phase are identical to those obtained for the initial phase in single turnover experiments (data not shown). They reflect the rate of $P_{i}$ release during the first turnover, before a later step in the reaction cycle becomes rate limiting. The reduction in the amplitude of the burst phase that is observed in the presence of PBP is most likely the consequence of the combined effects of reductions in the rates of ATP binding and hydrolysis. The linear phase corresponds to steady-state turnover of ATP and its duration increases proportional to the molar excess of ATP over myosin used. In the presence of 0 and $50 \mu \mathrm{M}$ PBP, the rates determined for the steady state phase correspond to 0.13 and $0.05 \mathrm{~s}^{-1}$, respectively. This suggests that steady-state turnover of ATP by actomyosin- $5 b$ is limited by ADP release and rebinding of ADP, as ADP competes efficiently with ATP for the actomyosin-5b binding site under the experimental conditions used. PBP appears to slow turnover further by increasing the affinity of $D d$ myosin-5b for ADP in the presence of actin. The third phase is governed by ATP depletion and accumulation of ADP. As a result, the efficiency of ADP to compete with ATP increases as phase three progresses. Additional support for a PBP-mediated increase in ADP affinity is provided by the reduced amplitudes of phases two and three in the presence of the inhibitor. (e) ATP induced dissociation of the complex formed by pyrene-labeled Factin and Dictyostelium myosin-5b in the absence and presence of PBP. $1 \mu \mathrm{M}$ pyracto•myosin complex were incubated with $5 \mu \mathrm{M}$ ATP for $10 \mathrm{~min}$ and the complex was dissociated by $0.5 \mathrm{mM}$ ATP in the absence and presence of $50 \mu \mathrm{M}$ PBP. The transients follow double exponential functions. In the absence of PBP, the following amplitudes $\left(\mathrm{A}_{\text {fast }}, \mathrm{A}_{\text {slow }}\right)$ and rate constants $\left(k_{\text {fast }}, k_{\text {slow }}\right)$ were obtained for the fast and slow phases: $A_{\text {fast }}=1.7, k_{\text {fast }}=46.7 \mathrm{~s}^{-1}, A_{\text {slow }}=0.8, k_{\text {slow }}=9.8 \mathrm{~s}^{-1}$. The following values were obtained in the presence of $50 \mu \mathrm{M}$ PBP: $A_{\text {fast }}=1.13, k_{\text {fast }}=11.1 \mathrm{~s}^{-1}, A_{\text {slow }}=1.08$, $k_{\text {slow }}=0.9 \mathrm{~s}^{-1}$. (f) ADP inhibition of ATP induced dissociation of the actomyosin-5b complex. Monophasic dissociation reactions were observed as the complex was dissociated with $1 \mathrm{mM}$ ATP in the presence of saturating ADP concentrations. The observed rate constants correspond directly to the ADP dissociation rate from AMD $\left(\mathbf{k}_{+4},=17.4 \mathrm{~s}^{-1}\right)$ or IAMD $\left(\mathbf{k}_{+4 \mathrm{i}}=7.0 \mathrm{~s}^{-1}\right)$. 

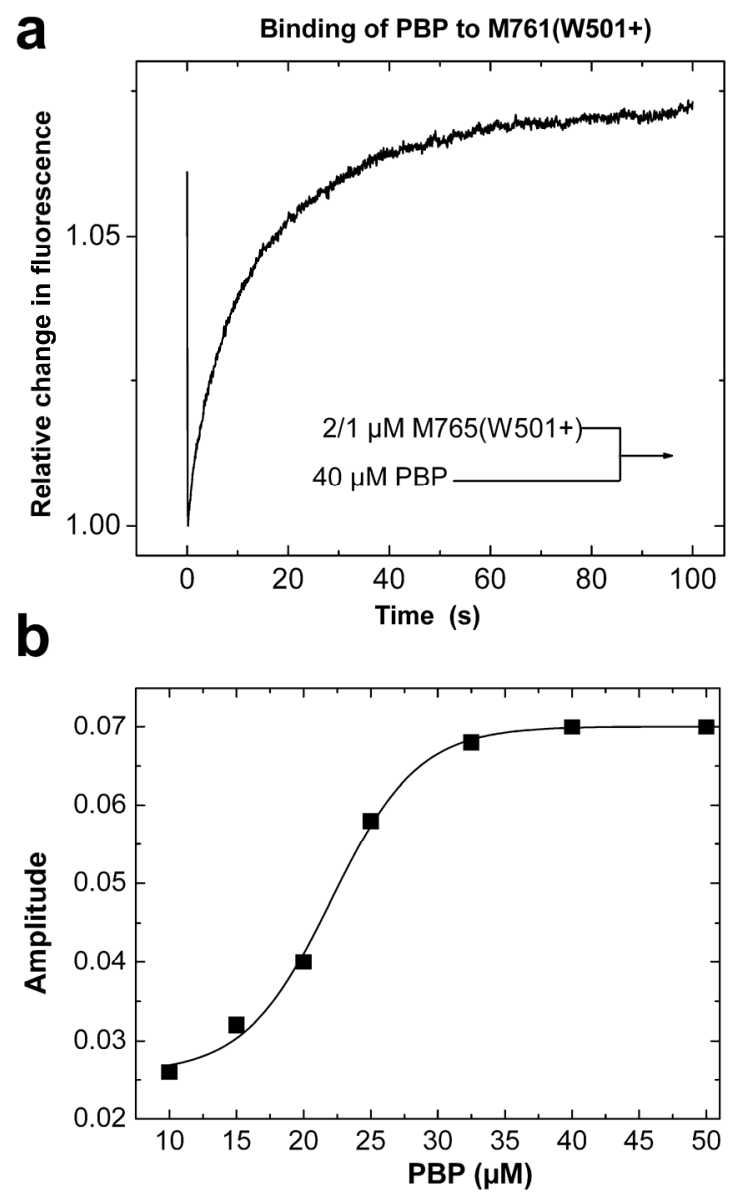

Supplemental Figure 2 PBP binding to myosin. (a) Fluorescence change on $40 \mu \mathrm{M}$ PBP binding to $1 \mu \mathrm{M} \mathrm{M765(Trp501+)} \mathrm{in} \mathrm{the} \mathrm{stopped-flow} \mathrm{fluorometer.} \mathrm{(b)}$ Dependence of the amplitudes of the slow exponential increase in fluorescence on PBP concentration. A sigmoidal fit of the observed change in the amplitudes plotted against the PBP concentration gives an apparent dissociation equilibrium constant $\left(K_{5 i}\right)$ for PBP binding to myosin-2 of $22.1 \mu \mathrm{M}$. To monitor PBP binding to myosin directly, we used a $D d$ myosin-2 motor domain construct retaining a single tryptophan residue at position 501 (Trp501) ${ }^{1}$. The fluorescence emission intensity from Trp501, located at the distal end of the relay helix, is very sensitive to the open-closed transition of the nucleotide binding pocket ${ }^{2,3}$. Mixing of PBP with the mutant myosin construct M765(Trp501+) resulted in a fast quenching of fluorescence followed by a slow recovery of the fluorescence signal. The observed process for the fluorescence recovery phase can be fitted to a single exponential function, and the observed rate constants display a linear dependence on PBP concentrations in the range of 10-50 $\mu \mathrm{M}$. A linear fit to the plot of the rates against PBP concentration results in an

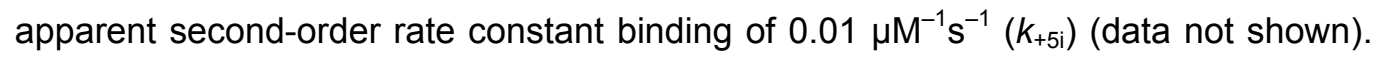
Fluorescence was excited at $296 \mathrm{~nm}$ and emission observed through a WG320 filter. 


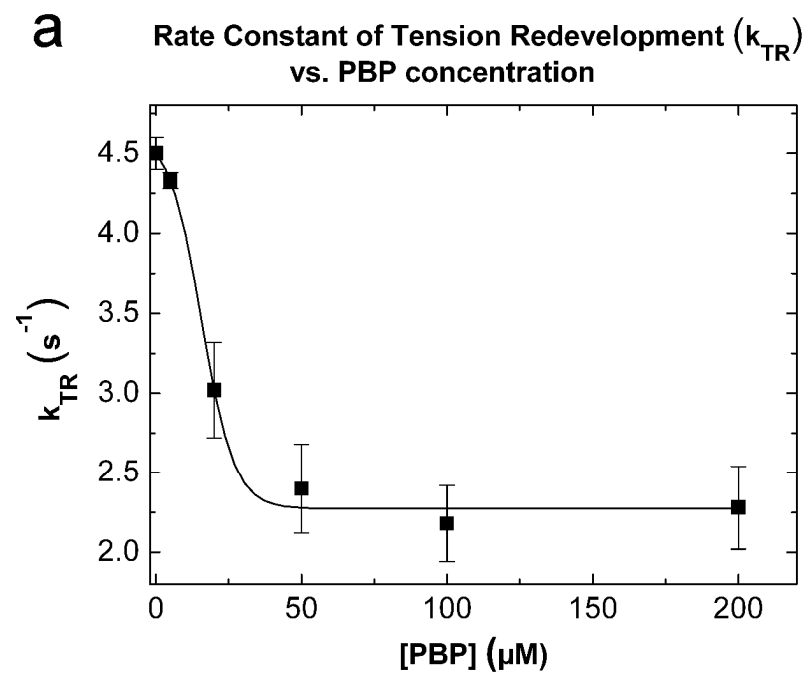

\section{b stiffness-speed profiles of relaxed fiber stiffness}

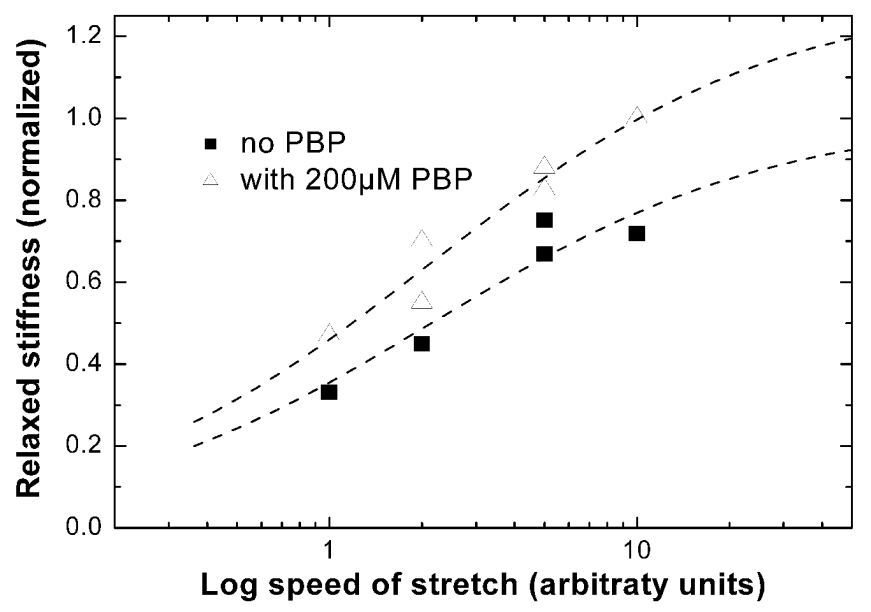

Supplemental Figure 3 PBP-mediated inhibition of muscle contraction. (a) PBPdependence of the rate constant of force redevelopment. The observed rate constant of tension redevelopment $\left(\mathrm{k}_{\mathrm{TR}}\right)$ is 2 -fold reduced in the presence of $100 \mu \mathrm{M}$ PBP. The change in $\mathrm{k}_{\mathrm{TR}}$ shows a sigmoidal dependence on the PBP concentration and gives an apparent $I_{50}$ value of $21 \mu \mathrm{M}$. (b) Measurements of stiffness-speed profiles in the absence and presence of PBP show small changes in dissociation rate or stiffness. The raise in stiffness observed upon addition of PBP was not greater than $20 \%$. In comparison, the addition of $\mathrm{Mg}^{2+}$-pyrophosphate increases fiber stiffness by 2 or 3 orders of magnitude, due to an increase in the number of strongly bound crossbridges $^{4}$. 
a

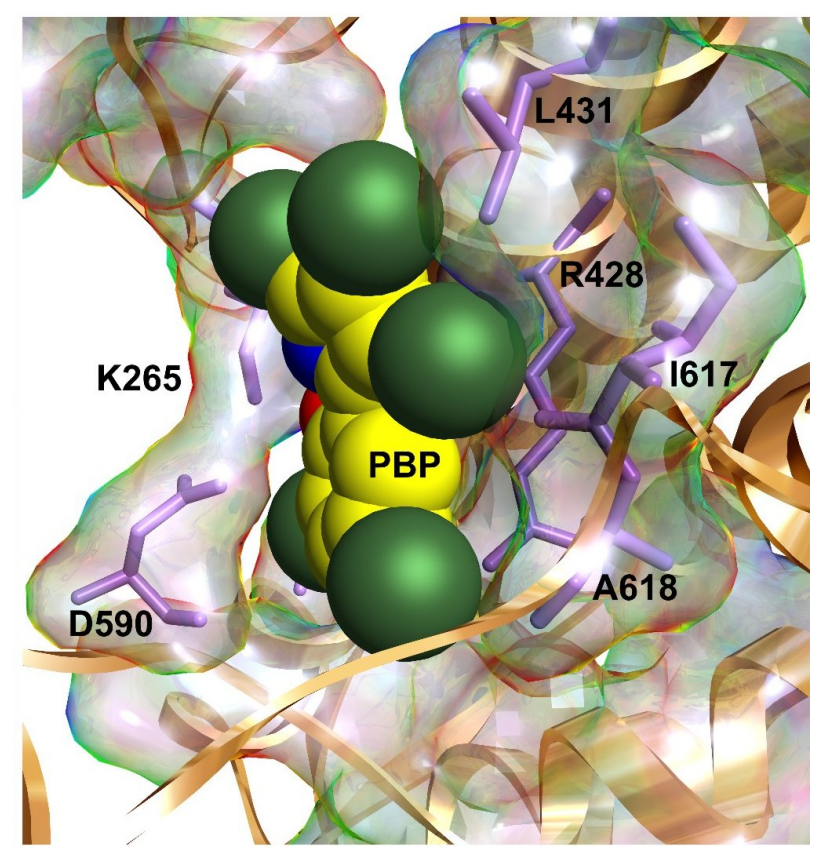

b

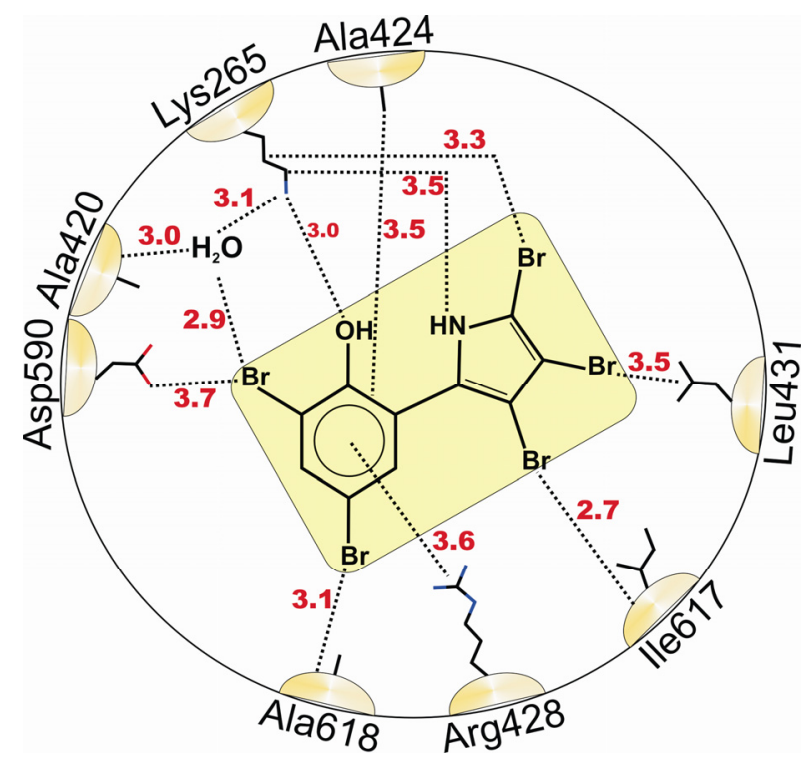

Supplemental Figure $4 D d$ myosin-2 PBP binding pocket. (a) Close-up view of the PBP binding pocket of $D d$ myosin-2. PBP is shown in spheres mode. (b) Schematic representation of interactions in the PBP binding pocket. Binding of PBP requires several rearrangements of residues inside and near the binding pocket. The most extensive of these changes involve residue Lys265. To allow PBP to bind, the ammonium group of Lys 265 needs to move $3.4 \AA$ towards Lys 423 . The movement of Lys265 involves a change in rotamer and the stabilization of the side chain in its new 
position by an intricate network of interactions. These include the formation of a key hydrogen bond with the hydroxyl group of the PBP phenol ring, a second hydrogen bond that is mediated via a water molecule from the ammonium group of Lys265 to the 2-bromo group of the phenyl ring, and contacts with the $\mathrm{NH}$-group and the 2bromo group of the pyrrole ring. Further stabilization is derived from a salt-bridge that forms between the ammonium group of Lys265 and the carboxyl group of Asp590. Formation of this salt-bridge requires a shift in the $\mathrm{C} \alpha$-backbone at position Asp590. The side chains of Ala424, Arg428, and Asp590 and the main chain carbonyl of Ala420 and Ala618 are involved in the binding of the phenyl ring, whereas the pyrrole ring has additional interactions with the side chain of Leu431 and the main chain carbonyl of lle617. The carbonyl groups of Ile617 and Ala618 are in hydrogenbonding distance to the bromo substituents in positions 3 of the pyrrole ring and 4 of the phenyl ring, while the hydrogen bond between the carbonyl group of Ala420 and the 2-bromo group of the phenyl ring is mediated by the same water molecule as the hydrogen bond between Lys265 and the hydroxyl group. The extent of side chain rearrangements associated with these interactions varies from $0.8 \AA$ for Leu431 to $3.5 \AA$ for Arg428. Residues Arg620 and Gln633 are not in contact with PBP but their side chains undergo coordinated conformational changes, bringing them in positions where they form part of the large opening of the pocket. The guanidinium group of Arg620 moves $3.5 \AA$ due to a $90^{\circ}$ rotation around the $\mathrm{C} \delta-\mathrm{C} \varepsilon$ bond, while a rotation around the $\mathrm{C} \alpha-\mathrm{C} \beta$ bond leads to a $1.5 \AA$ shift in the position of the side chain of Gln633. Notably, there are no residues from the L50 involved in PBP binding and the interactions with the strut-loop and loop-2 do not lead to perceptible changes in the L50. 


\section{Supplementary Methods}

ATP Binding and Hydrolysis Kinetics. Binding of ATP was modeled as a two-step process with initial formation of a binary collision complex followed by an ATPinduced conformational change. $D d$ myosin-5b lacks tryptophan residues close to the nucleotide binding pocket. As a consequence it shows no enhancement of intrinsic protein fluorescence associated with the initial nucleotide binding step and the observed $8 \%$ increase in intrinsic protein fluorescence that follows the addition of excess ATP monitors only the rate of the conformational change. Fluorescence transients were well described by a single exponential function. The observed rate constants show a linear dependence on the ATP concentration in the range from 2 to $25 \mu \mathrm{M}$ ATP. The apparent second-order rate constant for ATP binding is defined by the slope. At higher ATP concentrations the observed rate constants $k_{\text {obs }}$ are well described by a hyperbola $\left.\left(k_{\mathrm{obs}}=k_{\max }[\mathrm{ATP}] /(\mathrm{ATP}]+K_{0.5}\right)\right)$. At saturating ATP concentrations, $k_{\max }$ defines the maximum rate of the conformational change that corresponds to the rate of ATP hydrolysis in the absence of actin.

\section{Supplementary References}

1. Malnasi-Csizmadia, A., Kovacs, M., Woolley, R.J., Botchway, S.W. \& Bagshaw, C.R. The dynamics of the relay loop tryptophan residue in the Dictyostelium myosin motor domain and the origin of spectroscopic signals. J. Biol. Chem. 276, 19483-19490 (2001).

2. Batra, R. \& Manstein, D.J. Functional characterisation of Dictyostelium myosin II with conserved tryptophanyl residue 501 mutated to tyrosine. Biol. Chem. 380, 1017-1023 (1999).

3. Geeves, M.A., Fedorov, R. \& Manstein, D.J. Molecular mechanism of actomyosin-based motility. Cell. Mol. Life Sci. 62, 1462-1477 (2005).

4. Brenner, B., Yu, L.C., Greene, L.E., Eisenberg, E. \& Schoenberg, M. Ca ${ }^{2+}-$ sensitive cross-bridge dissociation in the presence of magnesium pyrophosphate in skinned rabbit psoas fibers. Biophys. J. 50, 1101-1108 (1986). 